\title{
Comparison of clinical manifestation, diagnosis and outcomes of invasive pulmonary aspergillosis and pulmonary mucormycosis
}

chun yu lin

Chang Gung Memorial Hospital

I-Ting Wang

Mackay Memorial Hospital

Che-Chia Chang

Chiayi Chang Gung Memorial Hospital

Wei-Chun Lee

Chiayi Chang Gung Memorial Hospital

Wei-Lun Liu

Fu Jen Catholic University Hospital

Yu-Chen Huang

Chang Gung Memorial Hospital

Ko-Wei Chang

Chang Gung Memorial Hospital

Hung-Yu Huang

Chang Gung Memorial Hospital

Hsuan-Ling Hsiao

Chang Gung Memorial Hospital

Kuo-chin Kao

Chang Gung Memorial Hospital

Chung-Chi Huang ( $\square$ cch4848@cloud.cgmh.org.tw)

George Dimopoulos

National and Kapodistrian University of Athens

Research article

Keywords: outcomes, diagnosis, invasive pulmonary aspergillosis, invasive pulmonary mucormycosis

Posted Date: August 25th, 2019

DOI: https://doi.org/10.21203/rs.2.13573/v1 
License: (c) (i) This work is licensed under a Creative Commons Attribution 4.0 International License. Read Full License

Version of Record: A version of this preprint was published at Microorganisms on November 5th, 2019. See the published version at https://doi.org/10.3390/microorganisms7110531. 


\section{Abstract}

Objects Invasive pulmonary mold infection usually has devastating outcomes. Timely differentiation between invasive pulmonary aspergillosis (IPA) from pulmonary mucormycosis (PM) is critical for treatment decision-making. However, information on IPA and PM differentiation is limited. Methods We conducted a retrospective, multicenter, observational study, with proven and probable IPA and PM patients from January 2004 to December 2017. Demographics, clinical manifestations, image reports, histopathological findings and outcomes were analyzed. Results A total of 47 IPA (34 proven and 13 probable) and $22 \mathrm{PM}$ (21 proven and 1 probable) cases were analyzed. The majority of tissues $(80 \%$ in IPA and $67 \%$ in PM) were obtained using bronchoscopy. While influenza infection was independently correlated to IPA, prior voriconazole exposure was independently associated with PM $(p=0.036, p=0.043$, respectively). The positive culture rate for PM was lower than that for IPA ( $41 \%$ vs. $69 \%, p=0.0363)$. The galactomannan (GM) level from serum and bronchoalveolar lavage (BAL) fluid was higher in IPA than in PM ( $3.1 \pm 0.5$ vs $0.7 \pm 0.6, p=0.0313 ; 4.3 \pm 0.6$ vs. $1.5 \pm 1.0, p=0.0375$, respectively). The overall mortality rate was $64 \%$, which was similar among IPA and PM groups. Systemic steroid exposure and high Acute Physiology and Chronic Health Evaluation II (APACHE II) scores on admission were independently correlated to mortality in IPA $(p=0.024)$, and concurrent bacterial sepsis predicted the in-hospital mortality among PM patients $(p=0.029)$. Conclusions Influenza infection and prior exposure to voriconazole may help physicians to differentiate IPA and PM. Bronchoscopy-guided biopsy and lavage specimen provide timely and definite diagnosis. While the prognosis of IPA is associated with systemic steroid exposure and higher APACHE II scores on admission, concurrent bacterial sepsis is the only independent predictor for mortality in PM.

\section{Background}

The incidence of invasive pulmonary mold infection has increased rapidly in recent years and is not limited to immunocompromised patients [1-3]. Patients with influenza infection with a critically ill status, diabetes mellitus, chronic lung disease, chronic renal failure, and liver cirrhosis may also develop invasive pulmonary fungal infections [2, 4-6]. Aspergillus spp. is the most common pathogen, followed by Mucorales [7]. Halo sign or air-crescent sign in chest computed tomography (CT) is the typical presentation in invasive pulmonary mold infection in neutropenic patients, but it has low sensitivity among non-neutropenic patients [8, 9]. Both invasive pulmonary aspergillosis (IPA) and pulmonary mucormycosis (PM) have devastating outcomes; hence, early and timely distinguishing IPA from PM are quite important for deciding the different treatment strategies [10]. However, accurate diagnosis is challenging for the identical presentations [1]. To our knowledge, there were only two studies discussing about the differentiation IPA from PM [10, 11]. Chamilo et al. evaluated 29 IPA (eight had definite IPA, 21 had probable IPA) and 16 PM (nine had definite PM and seven had probable PM) patients with cancer and found that concomitant sinusitis and voriconazole prophylaxis were predictors for PM [10]. They also showed that multiple nodules and pleural effusion on initial CT scan were independently associated with PM [10]. Jung et al. studied 96 IPA (12 had proven IPA, 84 had probable IPA) and 24 PM (20 had definite 
PM and four had probable PM) patients, mainly with hematologic malignancy and who were receiving solid organ/stem cell transplantation, and found that reverse halo sign was more common in PM and airway invasiveness was the feature of IPA in CT scans [11]. However, the differentiation of IPA from PM in clinical manifestation and CT findings was not conclusive and the outcome analysis was lacking in relatively immuno-competent patients. The current study aimed were to identify the differences in clinical manifestation, diagnostic approach of IPA and PM, and to analyze their outcomes.

\section{Methods}

\section{Study population}

This retrospective, multicenter, observational study was conducted from January 2004 to December 2017 at the Linkou and Chiayi branches of Chang Gung Memorial Hospital, Far Eastern Memorial Hospital, and the Liouying branch of Chi Mei Medical Center. According to the revised definitions for invasive fungal infections from the European Organization for the Research and Treatment of Cancer/ Mycosis Study Group (EORTC/MSG)[12], patients who met the criteria for proven or probable IPA or PM were included.

\section{Definitions and Classifications}

The diagnosis of proven invasive fungal infection is made using histopathology with evidence of filamentous fungi and tissue invasion. Probable invasive fungal infection referred to the presence of a positive culture for fungal species or positive GM from BAL in specific hosts with classic image manifestations. In patients with fungal tracheobronchitis, the image findings were classified into pseudomembranous, ulcerative, and obstructive forms according to Denning's classification [13].

Baseline characteristics, underlying conditions, laboratory parameters, image, and bronchoscopic findings, treatments, and outcomes were recorded. In-hospital mortality was accessed and the causes of death were also analyzed.

Informed consent was waived because this was a retrospective study and there was no modification in patient management. This study was approved by the institutional review boards of Chang Gung Memorial Hospital (CGMH 104-7452B).

\section{Statistical analysis}

Categorical variables were described using counts (percentages) and continuous variables as means \pm standard deviation (SD). We used Fisher's exact test for categorical variables and the student's $t$ test for continuous variables. Independent predicting factors for in-hospital mortality were determined using multivariate logistic regression analysis. All analyses were two-sided, and $p \llbracket 0.05$ was considered statistically significant. Statistical analyses were performed using Prism version 5 (GraphPad Software Inc., La Jolla, CA, USA) 


\section{Results}

\section{Patients' characteristics}

This study included 47 IPA ( 34 proven IPA and 13 probable IPA) and 22 PM patients (21 with proven PM and one with probable PM, Figure 1). Among these patients, 19 IPA patients and eight PM patients were reported in our published article [4]. There were four patients having concomitant IPA and PM, of which three were categorized as PM and one as IPA by histopathology. Thirty-two (68\%) patients with IPA had positive culture report from sputum, bronchial lavage fluid or tissue, and only nine (41\%) patients with PM had positive culture results $(p=0.0363$, Figure 1 , Table 1$)$.

Table 1 demonstrated the comparison of baseline manifestations between IPA and PM. Age, sex, smoking history, underlying disease, sinusitis, and steroid exposure were not significantly different between groups. However, in patients following influenza infection, who developed IPA were independently higher than PM (nine of $47,19 \%$ vs. $0 \%, p=0.0489$ ). Moreover, there were $14 \%$ patients (three of 22) who developed PM had prior voriconazole exposure which was independently higher than IPA (None had voriconazole exposure, $p=0.0321$ ). Forty (85\%) IPA patients and 19 (86\%) PM patients had received bronchoscopies and they had similar incidence of airway involvement (68\% in IPA and $73 \%$ in PM, respectively, Table 1). In both IPA and PM, pseudomembranous form was the most frequent seen subtype (66\% in IPA and $69 \%$ in PM), followed by ulcerative form (56\% in IPA and $69 \%$ in PM) and obstructive form (25\% in IPA and 13\% in PM). There were 34 (72\%) patients were proven IPA and 21 (95\%) patients were proven PM. Others were probable cases. Histologic finding of IPA and PM were demonstrated in Figure 2. In these proven cases, the tissues were mainly obtained from bronchoscopies ( $80 \%$ in IPA and $67 \%$ in PM), followed by surgery and CT-guided biopsy. None of these patients developed massive hemoptysis or irreversible hypoxemia after procedures. Most of the fungal cultures were obtained using bronchoscopy in both IPA (78\%) and PM (78\%). However, the PM patients had a significantly higher negative culture rate versus IPA ( 13 of $22,59 \%$ vs. 15 of $47,32 \%, p=0.0363$ ). The level of GM from serum and BAL were significantly higher in IPA patients than in PM patients $(3.2 \pm 0.5 \mathrm{vs} 0.7$ $\pm 0.6, p=0.0259 ; 4.1 \pm 0.6$ vs. $1.5 \pm 1.0, p=0.0435)$. In patients who were exposed to a CT scan $(74 \%$ in IPA and $91 \%$ in PM), consolidation was the most frequent finding in both group (83\% in IPA and 95\% in PM). There were four patients that had only airway involvement and all of them were IPA, but the difference was not significant versus PM patients $(p=0.2848)$. Comparing to IPA, PM was associated with more abscess formation ( 3 of $47,9 \%$ vs. 7 of $22,35 \%, p=0.0301$ ). But the difference was not significant in multivariate analysis. Patients with IPA had higher APACHE II scores on admission (18.5 \pm 1.4 vs.13.3 \pm $1.7, p=0.0243)$. There were 30 (68\%) IPA patients and 14 (64\%) PM patients developed respiratory failure before diagnosis. More PM patients receiving polyene treatment at diagnosis (five, $23 \%$ vs. one, $2 \%$, $p=0.0126$ ) and surgical interventions (seven, $35 \%$, five, $11 \%, p=0.0488$ ).

There were 45 death, 44 patients died from invasive fungal pneumonia. One had IPA with good response to voriconazole treatment, but died of brain stem ischemic stroke. Nonetheless, the in-hospital mortality because of invasive fungal pneumonia were similar in IPA and PM $(29,62 \%$ vs. $15,68 \%, p=1.0)$. 
Predicting factors for in-hospital mortality were summarized in Table 2. Survival was not related to underlying disease in both IPA and PM (data not shown). In univariate analysis, systemic steroid exposure, higher APACHE II score on admission, respiratory failure before diagnosis and surgical intervention were significant predicting factors for in-hospital mortality in IPA. However, in multivariate analysis, systemic steroid exposure and higher APACHE II score on admission were the independent prognostic factor in IPA ( $p=0.024 ; p=0.024$, Table 2$)$. In PM, only concurrent bacterial sepsis was the only independent predictor for in-hospital mortality $(p=0.029)$.

\section{Discussion}

To the best of our knowledge, this report was the first one discussing the diagnostic approach and included the largest series of comparisons in clinical manifestations of patients with IPA and PM. Approximately half of our patients had DM and chronic lung disease. Only 11 patients (16\%) had hematologic disease and eight of these were under neutropenic status. Influenza infection was independently associated with IPA. Prior exposure to voriconazole provided clinical hint for diagnosing PM. While PM had lower culture rate comparing to IPA, the GM level from serum and BAL were significantly higher among IPA patients. Seventy-two percent of patients with IPA and $95 \%$ of patients with PM were proven cases and two third of the diagnosing tissues were obtained from bronchoscopy. The mortality was greater than $60 \%$ in both IPA and PM patients. Systemic steroid exposure and APACHE II score on admission were independently correlated to mortality in IPA, and concurrent bacterial sepsis independently predicted the in-hospital mortality among PM patients.

In recent decades, we found that not only immunocompromised patients were susceptible to fungal infections, diabetes mellitus, chronic lung diseases may also experience invasive fungal infections [4]. In one review article, Patterson et al. demonstrated that critically ill patients who are admitted to intensive care units (ICUs) also have higher risk of pulmonary fungal infection [2]. Wauters et al. revealed that IPA is a more frequent complication in critically ill H1N1 patients and suggest that use of systemic steroid in these patients is an independent risk factor for fungal infections [5]. Moreover, in one largest multicenter retrospective study, Schauwvlieghe et al. collected 457 critically ill patients with influenza comparing to 321 patients with community acquired pneumonia and demonstrated that influenza was independently associated with IPA [14]. In current study, DM and chronic lung disease was the most common underlying disease in both IPA and PM patients. The underlying disease was similar in IPA and PM. However, we found that after influenza infection, patients were independently more susceptible to IPA rather than PM ( $19 \%$ vs. $0 \%, p=0.036)$. IPA is the most common mold infection, followed by PM [1]. Nevertheless, the incidence of PM seems to rise in recent years $[4,7,15]$. Neofytos et al found that after bronchoscopy initiation, the observed rate of invasive mucormycosis among HSCT recipients increased from $0.6 \%$ annually to $3.0 \%$ [7]. In our previous report, $25 \%$ of bronchoscopy diagnosed invasive fungal tracheobronchitis were caused by Mucorales [4]. In current investigation, among these invasive pulmonary mold infection patients, one third of them had PM. Carol Garcia-Vidal et al. had proposed that voriconazole prophylaxis had significantly associated with zygomycosis in hematologic patients [16]. Moreover, in animal models, the virulence of zygomycytes may increase after exposure to voriconazole 
[17]. In current report, prior voriconazole exposure was strongly associated with PM (14\% vs. $0 \%$ ). Moreover, two of three patients had concomitant IPA and PM based on the histopathologic report was under voriconazole treatment before definite diagnosis. Although the exposure to voriconazole may not a directly leading to PM, the clinical awareness of PM should be risen when the patients had prior voriconazole exposure.

Typical findings in CT scan, such as halo sign (HS) for IPA, reverse halo sign (RHS) for PM and aircrescent sign, were useful in diagnosing invasive pulmonary mold infection in immunocompromised patients. In one review article, Georgiado et al. found lots of diseases other than invasive pulmonary mold infection presenting with those findings [8]. Nam et al. recently presented that consolidation or mass with halo sign were the most common finding on CT scan in PM. The serial morphologic changes into reverse halo sign, central necrotic cavity or air-crescent sign were noted after treatment and recovery of neutropenia [18]. However, articles discussing about CT finding in non-neutropenic patients were scarce. Jung et al. conducted a retrospective study focusing on comparison of CT scan in IPA and PM. They found the RHS was more common in PM. But the RHS was more frequent developed in neutropenic patients and half of them only appeared within five days from symptoms onset [11]. In our study, which mainly composed by non-neutropenic patients, the most common finding on CT scan was consolidation (29 of $33,83 \%$ in IPA vs. 19 of 20, 95\% in PM, in patients who receiving CT scan, respectively). There were a total 8 patients (12\%) were under neutropenia status while diagnosing invasive mold infections and five had consolidation in CT scan, one had merely airway invasion, one had cavitation and one had abscess formation. None of our patients had HS or RHS. In the CT scan findings among our invasive pulmonary mold infection patients, only abscess formation was higher in PM patients than IPA (35\% vs. 9\%, $p=0.028$ ), but it was not independently significant in multivariate analysis.

The fungal culture takes lots of time and positive rate in lower respiratory tract samples is 48 to $76 \%$ in IPA patients $[11,19]$ and ranged from $29 \%$ to $46 \%$ in PM patients $[11,20]$. In current report, the positive culture rate was significantly higher in IPA than in PM ( $68 \%$ vs. $41 \%$, respectively, $p=0.0363)$. Among these patients, most of the positive culture specimen were obtained from bronchoscopy (78\% in both IPA and PM). GM is a fungal cell wall component which is released during tissue invasion by Aspergillus hyphae and can be detected in serum and bronchoalveolar lavage (BAL) fluid [21]. Serum levels in nonneutropenic patients may be underestimate because circulating neutrophils are able to clear the antigen [22]. Although the value of serum GM can be influenced by neutrophil counts [22, 23], GM in BAL sample provides reliable diagnosing tools in IPA [21]. Moreover, GM had no role in diagnosing PM. In current study, we demonstrated that GM in both serum and BAL fluid were significantly higher in IPA than in PM ( $3.2 \pm 0.5$ vs $0.7 \pm 0.6, p=0.0259 ; 4.1 \pm 0.6$ vs. $1.5 \pm 1.0, p=0.0435)$. Considering the possible influence of neutrophil counts in serum GM, samples from BAL is a good choice for differentiate IPA from PM. Furthermore, in our research, 72\% (34 of 47) of IPA and 95\% (21 of 22) of PM were proven cases. Among them, $80 \%$ (28 of 34 ) proven IPA and 67\% (14 of 21) proven PM tissue were obtained from bronchoscopy. Moreover, none of these patients developed massive hemoptysis or major complications after bronchoscopy. Although we found that prior use of voriconazole was independently associated with PM, there were three patients had concomitant IPA and PM. Chamilos et al. also had found five cancer 
patients had concomitant IPA and PM [10]. Considering the concomitant IPA and PM, the precise and timely diagnosis may still mainly depend on histopathology. In patients with high risk of surgical intervention, biopsy from bronchoscopy and specimen from BAL for GM test and fungal culture may be an ideal diagnosing method.

Many studies reveal that underlying conditions, such as neutropenia, active malignancy, liver disease, hematopoietic stem cell or solid organ transplant, are risk factors for mortality in invasive mold infection [7, 24-26]. In current study, only 11 patients (16\%) had hematologic disease and eight were under neutropenic status. We found that underlying disease had no association with in-hospital mortality in both IPA and PM. In addition to uncontrolled underlying disease, Taccone et al. showed that mechanical ventilation, renal replacement therapy during ICU stay and higher Sequential Organ Failure Assessment score at diagnosis were independent predictors for death of invasive aspergillosis in critically ill patients [27]. Two third of our patients (68\% in IPA and $64 \%$ in PM) developed respiratory failure before diagnosis and had admitted to ICU. The usage of systemic steroid, respiratory failure before diagnosis, surgical intervention were correlated to mortality in IPA, but only systemic steroid exposure and high APACHE II score on admission were independent risk factor for in-hospital mortality among IPA patients. Regarding to PM, Lin et al. conducted a retrospective study which composed more than $70 \%$ patients had hematologic malignancy and were under neutropenic status. They demonstrated that concurrent bacteremia is the sole independent predictor for mortality among those PM patients [20]. In our PM patients, which mainly composed with underlying diabetes mellitus (DM) and chronic lung disease (59\% and $36 \%$, respectively), concurrent bacterial sepsis was still the only independent predictor for in-hospital mortality. These finding suggest that the outcomes of IPA and PM were more closely related to the clinical disease severity rather than underlying conditions.

The present study has some limitations. First, for the retrospective nature of this study, the sample size was limited and some of the data are incomplete, this probably led to diminished generalizability; second, we only included proven and probable cases, patients with possible invasive mold infection may have been overlooked, and the outcomes may have been underestimated.

\section{Conclusions}

Differentiation for IPA and PM are difficult. Influenza infection and prior exposure to voriconazole may help physicians to identify IPA from PM and initiate different antifungal agent earlier. Bronchoscopy may be a safe and useful tool to obtain the specimen for fungal culture, GM test and histopathology, and provide more precise and timely diagnosis. The outcome of IPA was associated with systemic steroid exposure and higher APACHE II score on admission. Concurrent bacterial sepsis was the only independent predictor for in-hospital mortality in PM. Further larger, prospective studies are required.

\section{Declarations}

\section{Ethics approval and consent to participate}


The study was approved by the institutional review board of Chang Gung Memorial Hospital (CGMH 1047452B). Informed consent was waived because this was a retrospective study and there was no modification in patient management. All personal information was encrypted in the database, and patient data accessed was de-identified. There was no breach of privacy.

\section{Consent for publication}

Not applicable.

\section{Availability of data and material}

The datasets analyzed during the current study are available from the corresponding author upon reasonable request.

\section{Funding}

This research did not receive any specific grant from funding agencies in the public, commercial, or notfor-profit sectors.

\section{Conflict of interest}

The authors declare that they have no competing interests.

\section{Authors' contributions}

CYL, ITW, WLL, CCC, WCL, YCH, KWC, HYH, HLH, KCK, CCH, GD had conception and design of the study. CYL, ITW, YCH, KWC had drafted the manuscript. YCH, KWC, HYH, HLH, KCK had provided the study materials and patients. $\mathrm{CYL}, \mathrm{YCH}, \mathrm{KWC}, \mathrm{HYH}, \mathrm{HLH}$ had collected and assembled of data. CYL, CCH, GD had analyzed and interpreted data. WLL, CCC, WCL, HYH, HLH, KCK, CCH, GD had revised the manuscript.

All authors read and approved the final manuscript.

\section{Acknowledgments}

We thank all the investigators and members of the Department of Thoracic Care Medicine for their effort.

\section{References}

1. Limper AH, Knox KS, Sarosi GA, Ampel NM, Bennett JE, Catanzaro A, et al. An official American Thoracic Society statement: Treatment of fungal infections in adult pulmonary and critical care patients. Am J Respir Crit Care Med. 2011;183(1):96-128. doi: 10.1164/rccm.2008-740ST. PubMed PMID: 21193785.

2. Patterson KC, Strek ME. Diagnosis and treatment of pulmonary aspergillosis syndromes. Chest. 2014;146(5):1358-68. doi: 10.1378/chest.14-0917. PubMed PMID: 25367472. 
3. Bassetti M, Bouza E. Invasive mould infections in the ICU setting: complexities and solutions. $J$ Antimicrob Chemother. 2017;72(suppl_1):i39-i47. doi: 10.1093/jac/dkx032. PubMed PMID: 28355466.

4. Lin CY, Liu WL, Chang CC, Chang HT, Hu HC, Kao KC, et al. Invasive fungal tracheobronchitis in mechanically ventilated critically ill patients: underlying conditions, diagnosis, and outcomes. Ann Intensive Care. 2017;7(1):9. doi: 10.1186/s13613-016-0230-9. PubMed PMID: 28083768; PubMed Central PMCID: PMCPMC5233606.

5. Wauters J, Baar I, Meersseman P, Meersseman W, Dams K, De Paep R, et al. Invasive pulmonary aspergillosis is a frequent complication of critically ill H1N1 patients: a retrospective study. Intensive Care Med. 2012;38(11):1761-8. doi: 10.1007/s00134-012-2673-2. PubMed PMID: 22895826.

6. Blot SI, Taccone FS, Van den Abeele AM, Bulpa P, Meersseman W, Brusselaers N, et al. A clinical algorithm to diagnose invasive pulmonary aspergillosis in critically ill patients. Am J Respir Crit Care Med. 2012;186(1):56-64. doi: 10.1164/rccm.201111-19780C. PubMed PMID: 22517788.

7. Neofytos D, Treadway S, Ostrander D, Alonso CD, Dierberg KL, Nussenblatt V, et al. Epidemiology, outcomes, and mortality predictors of invasive mold infections among transplant recipients: a 10year, single-center experience. Transpl Infect Dis. 2013;15(3):233-42. doi: 10.1111/tid.12060. PubMed PMID: 23432974; PubMed Central PMCID: PMCPMC3664270.

8. Georgiadou SP, Sipsas NV, Marom EM, Kontoyiannis DP. The diagnostic value of halo and reversed halo signs for invasive mold infections in compromised hosts. Clin Infect Dis. 2011;52(9):1144-55. doi: 10.1093/cid/cir122. PubMed PMID: 21467021; PubMed Central PMCID: PMCPMC3106265.

9. Dai Z, Zhao H, Cai S, Lv Y, Tong W. Invasive pulmonary aspergillosis in non-neutropenic patients with and without underlying disease: a single-centre retrospective analysis of 52 subjects. Respirology. 2013;18(2):323-31. doi: 10.1111/j.1440-1843.2012.02283.x. PubMed PMID: 23051143.

10. Chamilos G, Marom EM, Lewis RE, Lionakis MS, Kontoyiannis DP. Predictors of pulmonary zygomycosis versus invasive pulmonary aspergillosis in patients with cancer. Clin Infect Dis. 2005;41(1):60-6. doi: 10.1086/430710. PubMed PMID: 15937764.

11. Jung J, Kim MY, Lee HJ, Park YS, Lee SO, Choi SH, et al. Comparison of computed tomographic findings in pulmonary mucormycosis and invasive pulmonary aspergillosis. Clin Microbiol Infect. 2015;21(7):684 e11-8. doi: 10.1016/j.cmi.2015.03.019. PubMed PMID: 25882362.

12. De Pauw B, Walsh TJ, Donnelly JP, Stevens DA, Edwards JE, Calandra T, et al. Revised definitions of invasive fungal disease from the European Organization for Research and Treatment of Cancer/Invasive Fungal Infections Cooperative Group and the National Institute of Allergy and Infectious Diseases Mycoses Study Group (EORTC/MSG) Consensus Group. Clin Infect Dis. 2008;46(12):1813-21. doi: 10.1086/588660. PubMed PMID: 18462102; PubMed Central PMCID: PMCPMC2671227.

13. Denning DW. Commentary: unusual manifestations of aspergillosis. Thorax. 1995;50(7):812-3. PubMed PMID: 7570425; PubMed Central PMCID: PMCPMC474663. 
14. Schauwvlieghe A, Rijnders BJA, Philips N, Verwijs R, Vanderbeke L, Van Tienen C, et al. Invasive aspergillosis in patients admitted to the intensive care unit with severe influenza: a retrospective cohort study. Lancet Respir Med. 2018;6(10):782-92. doi: 10.1016/S2213-2600(18)30274-1. PubMed PMID: 30076119.

15. Abidi MZ, Sohail MR, Cummins N, Wilhelm M, Wengenack N, Brumble L, et al. Stability in the cumulative incidence, severity and mortality of 101 cases of invasive mucormycosis in high-risk patients from 1995 to 2011: a comparison of eras immediately before and after the availability of voriconazole and echinocandin-amphotericin combination therapies. Mycoses. 2014;57(11):687-98. doi: 10.1111/myc.12222. PubMed PMID: 25040241; PubMed Central PMCID: PMCPMC4192082.

16. Garcia-Vidal C, Upton A, Kirby KA, Marr KA. Epidemiology of invasive mold infections in allogeneic stem cell transplant recipients: biological risk factors for infection according to time after transplantation. Clin Infect Dis. 2008;47(8):1041-50. doi: 10.1086/591969. PubMed PMID: 18781877; PubMed Central PMCID: PMCPMC2668264.

17. Lamaris GA, Ben-Ami R, Lewis RE, Chamilos G, Samonis G, Kontoyiannis DP. Increased virulence of Zygomycetes organisms following exposure to voriconazole: a study involving fly and murine models of zygomycosis. J Infect Dis. 2009;199(9):1399-406. doi: 10.1086/597615. PubMed PMID: 19358672.

18. Nam BD, Kim TJ, Lee KS, Kim TS, Han J, Chung MJ. Pulmonary mucormycosis: serial morphologic changes on computed tomography correlate with clinical and pathologic findings. Eur Radiol. 2018;28(2):788-95. doi: 10.1007/s00330-017-5007-5. PubMed PMID: 28812135.

19. Bulpa P, Dive A, Sibille Y. Invasive pulmonary aspergillosis in patients with chronic obstructive pulmonary disease. Eur Respir J. 2007;30(4):782-800. doi: 10.1183/09031936.00062206. PubMed PMID: 17906086.

20. Lin E, Moua T, Limper AH. Pulmonary mucormycosis: clinical features and outcomes. Infection. 2017;45(4):443-8. doi: 10.1007/s15010-017-0991-6. PubMed PMID: 28220379.

21. Meersseman W, Lagrou K, Maertens J, Wilmer A, Hermans G, Vanderschueren S, et al. Galactomannan in bronchoalveolar lavage fluid: a tool for diagnosing aspergillosis in intensive care unit patients. Am J Respir Crit Care Med. 2008;177(1):27-34. doi: 10.1164/rccm.200704-6060C. PubMed PMID: 17885264.

22. Bassetti M, Righi E, De Pascale G, De Gaudio R, Giarratano A, Mazzei T, et al. How to manage aspergillosis in non-neutropenic intensive care unit patients. Crit Care. 2014;18(4):458. doi: 10.1186/s13054-014-0458-4. PubMed PMID: 25167934; PubMed Central PMCID: PMCPMC4220091.

23. Cordonnier C, Botterel F, Ben Amor R, Pautas C, Maury S, Kuentz M, et al. Correlation between galactomannan antigen levels in serum and neutrophil counts in haematological patients with invasive aspergillosis. Clin Microbiol Infect. 2009;15(1):81-6. doi: 10.1111/j.14690691.2008.02122.x. PubMed PMID: 19154482.

24. Spellberg B, Kontoyiannis DP, Fredricks D, Morris MI, Perfect JR, Chin-Hong PV, et al. Risk factors for mortality in patients with mucormycosis. Med Mycol. 2012;50(6):611-8. doi: 
10.3109/13693786.2012.669502. PubMed PMID: 22435877; PubMed Central PMCID:

PMCPMC3951871.

25. Garcia-Vidal C, Peghin M, Cervera C, Gudiol C, Ruiz-Camps I, Moreno A, et al. Causes of death in a contemporary cohort of patients with invasive aspergillosis. PLoS One. 2015;10(3):e0120370. doi: 10.1371/journal.pone.0120370. PubMed PMID: 25803853; PubMed Central PMCID: PMCPMC4372359.

26. Nivoix Y, Velten M, Letscher-Bru V, Moghaddam A, Natarajan-Ame S, Fohrer C, et al. Factors associated with overall and attributable mortality in invasive aspergillosis. Clin Infect Dis. 2008;47(9):1176-84. doi: 10.1086/592255. PubMed PMID: 18808352.

27. Taccone FS, Van den Abeele AM, Bulpa P, Misset B, Meersseman W, Cardoso T, et al. Epidemiology of invasive aspergillosis in critically ill patients: clinical presentation, underlying conditions, and outcomes. Crit Care. 2015;19:7. doi: 10.1186/s13054-014-0722-7. PubMed PMID: 25928694; PubMed Central PMCID: PMCPMC4344741.

\section{Abbreviations}

IPA: invasive pulmonary aspergillosis, PM: pulmonary mucormycosis, GM: galactomannan, BAL: bronchoalveolar lavage, APACHE II: Acute Physiology and Chronic Health Evaluation II, CT: computed tomography, ICU: intensive care units, DM: diabetes mellitus, HS: halo sign, RHS: reverse halo sign.

\section{Tables}

Table 1. Comparison of clinical characteristics, treatments and outcomes between IPA and PM 


\begin{tabular}{|c|c|c|c|c|}
\hline & \multicolumn{3}{|c|}{ Univariate } & Multivariate \\
\hline & IPA $(n=47)$ & $\mathrm{PM}(\mathrm{n}=22)$ & $\mathrm{p}$ value & $\mathrm{p}$ value \\
\hline Age, years, mean \pm SD & $61.2 \pm 2.4$ & $59.0 \pm 3.9$ & 0.6182 & \\
\hline Sex, male, no. (\%) & $33(70)$ & 17 (77) & 0.5714 & \\
\hline Smoking history, no. (\%) & $21(45)$ & $14(64)$ & 0.1232 & \\
\hline
\end{tabular}

Underlying disease, no. (\%)

\footnotetext{
DM

Chronic lung disease

Solid organ cancer

Hematologic disease

Neutropenia

Cirrhosis

Organ Transplantation

Autoimmune disease

Sinusitis, no. (\%)

Systemic steroid, no. (\%)

Immunosuppressant, no. (\%)

Post Influenza, no. (\%)

Prior Antifungal agent, no. (\%)

Voriconazole

Polyene

Echinocandin

Bronchoscopy, no. (\%)

Airway involvement, no. (\%)

Scope pattern, no. (\%)

Pseudomembrane

Ulcerative

Obstructive
}

Diagnostic classification, no. (\%)

Proven

Probable

Proven cases, no. (\%)

Bronchoscopyc 28 (80)

Surgery 4 (11)

CT-guided biopsy 3 (9)

Negative fungal culture, no. (\%) 15 (32)

Positive fungal culture, no. (\%) 32 (68)

Bronchoscopy

Sputum

25 (78)

9 (28)

Tissue

Galatomannan

Serum

BAL

CT finding, no. (\%)

Consolidation

Cavitation

Abscess formation

Airway only

Ball in hole

APACHE II score on admission, mean \pm SD

$\mathrm{RF}$ before diagnosis, no. (\%)

Concurrent bacterial sepsis, no. (\%)

Antifungal therapy at diagnosis,
1 (3)

$3.2 \pm 0.5$

$4.1 \pm 0.6$

\begin{tabular}{|c|c|c|c|}
\hline $\begin{array}{l}23(49) \\
21(45)\end{array}$ & $\begin{array}{l}13(59) \\
8(36)\end{array}$ & $\begin{array}{l}0.4503 \\
0.4467\end{array}$ & \\
\hline 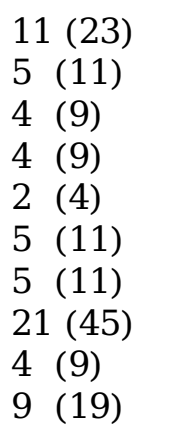 & $\begin{array}{ll}2 & (9) \\
6 & (27) \\
4 & (18) \\
2 & (9) \\
0 & (0) \\
0 & (0) \\
6 & (27) \\
7 & (32) \\
0 & (0) \\
0 & (0)\end{array}$ & $\begin{array}{l}0.1937 \\
0.1572 \\
0.4231 \\
1.0 \\
1.0 \\
0.1633 \\
0.2379 \\
0.2984 \\
0.2943 \\
0.0489\end{array}$ & 0.036 \\
\hline $\begin{array}{l}0 \quad(0) \\
2 \quad(4) \\
1 \quad(2) \\
40(85) \\
32(68)\end{array}$ & $\begin{array}{l}3(14) \\
0(0) \\
2(9) \\
19(86) \\
16(73)\end{array}$ & $\begin{array}{l}0.0321 \\
1.0 \\
0.2491 \\
1.0 \\
1.0\end{array}$ & 0.043 \\
\hline $\begin{array}{l}21(66) \\
18(56) \\
8(25)\end{array}$ & $\begin{array}{l}11(69) \\
11(69) \\
2(13) \\
21(95) \\
1(5)\end{array}$ & $\begin{array}{l}1.0 \\
0.5352 \\
0.4597 \\
0.2525\end{array}$ & \\
\hline & $\begin{array}{l}14(67) \\
6(29) \\
1(3) \\
13(59)\end{array}$ & $\begin{array}{l}0.3483 \\
0.1562 \\
1.0 \\
0.0363\end{array}$ & \\
\hline & $\begin{array}{ll}9 & (41) \\
7 & (78) \\
2 & (22)\end{array}$ & $\begin{array}{l}1.0 \\
1.0\end{array}$ & \\
\hline & $0(0)$ & 1.0 & \\
\hline & $\begin{array}{l}0.7 \pm 0.6 \\
1.5 \pm 1.0\end{array}$ & $\begin{array}{l}0.0259 \\
0.0435\end{array}$ & \\
\hline 35 (74) & $20(91)$ & & \\
\hline 29 (83) & 19 (95) & 0.2333 & \\
\hline $\begin{array}{ll}7 & (20) \\
3 & (9)\end{array}$ & $\begin{array}{ll}6 & (30) \\
7 & (35)\end{array}$ & $\begin{array}{l}0.5221 \\
0.0301\end{array}$ & 0.081 \\
\hline $4(11)$ & $0(0)$ & 0.2848 & \\
\hline $2(6)$ & 1 (5) & 1.0 & \\
\hline $\begin{array}{l}18.5 \pm 1.4 \\
32(68)\end{array}$ & $\begin{array}{l}13.3 \pm 1.7 \\
14(64)\end{array}$ & $\begin{array}{l}0.0243 \\
10\end{array}$ & \\
\hline $20(43)$ & $12(55)$ & 0.3029 & \\
\hline
\end{tabular}

no. (\%)

Ployene 
Voriconazole

Itraconazole

Caspofungin

Surgical intervention, no. (\%)

In-hospital Mortality of
19 (40)

6 (13)

5 (11)

5 (11)

29 (62)
5 (23)

1 (5)

2 (9)

7 (35)

15 (68)
0.2747

0.4116

1.0

0.0488

1.0

invasive mold pneumonia, no. (\%)

Abbreviation: SD: standard deviation; IPA: invasive pulmonary aspergillosis; PM: pulmonary mucormycosis; DM: diabetes mellitus; BAL: bronchoalveolar lavage fluid; CT: computer tomography; APACHE II: Acute Physiology and Chronic Health Evaluation II; RF: respiratory failure.

\section{Table 2}

Predicting factors for in-hospital mortality in IPA and PM. 


\begin{tabular}{|c|c|c|c|c|c|c|c|c|}
\hline & \multicolumn{4}{|c|}{ IPA } & \multicolumn{4}{|c|}{$\overline{P M}$} \\
\hline & \multicolumn{3}{|c|}{ Univariate } & \multirow{2}{*}{$\begin{array}{l}\text { Multivariate } \\
\mathrm{P} \text { value }\end{array}$} & \multicolumn{3}{|c|}{ Univariate } & \multirow{2}{*}{$\begin{array}{l}\text { Multivariate } \\
\text { P value }\end{array}$} \\
\hline & $\begin{array}{l}\text { Survivor } \\
\mathrm{N}=18\end{array}$ & $\begin{array}{l}\text { Non- } \\
\text { Survivor } \\
\mathrm{N}=29\end{array}$ & $\mathrm{P}$ value & & $\begin{array}{l}\text { Survivor } \\
\mathrm{N}=7\end{array}$ & $\begin{array}{l}\text { Non- } \\
\text { Survivor } \\
\mathrm{N}=15\end{array}$ & $\begin{array}{l}\mathrm{P} \\
\text { value }\end{array}$ & \\
\hline $\begin{array}{l}\text { Systemic steroid, } \\
\text { no. (\%) }\end{array}$ & 3 & 18 & 0.0029 & 0.024 & 1 & 6 & 0.3501 & \\
\hline $\begin{array}{l}\text { Post Influenza, } \\
\text { no. (\%) }\end{array}$ & 2 & 6 & 0.6918 & & 0 & 0 & 1.0 & \\
\hline $\begin{array}{l}\text { Neutropenia, no. } \\
\text { (\%) }\end{array}$ & 1 & 3 & 1.0 & & 1 & 3 & 1.0 & \\
\hline $\begin{array}{l}\text { APACHE II score } \\
\text { on admission, } \\
\text { mean+SD }\end{array}$ & $13.2 \pm 1.6$ & $21.5 \pm 1.7$ & 0.0024 & 0.024 & $8.6 \pm 1.4$ & $15.5 \pm 2.1$ & 0.0463 & 0.99 \\
\hline $\begin{array}{l}\text { RF before } \\
\text { diagnosis, no. } \\
\text { (\%) }\end{array}$ & 6 & 26 & $<0.0001$ & 0.460 & 2 & 12 & 0.0524 & 1.0 \\
\hline $\begin{array}{l}\text { Concurrent } \\
\text { bacterial sepsis, } \\
\text { no. (\%) }\end{array}$ & 5 & 15 & 0.1366 & & 1 & 11 & 0.0201 & 0.029 \\
\hline $\begin{array}{l}\text { Airway } \\
\text { involvement, no. } \\
\text { (\%) }\end{array}$ & 10 & 22 & 0.4935 & & 4 & 12 & 0.3341 & \\
\hline $\begin{array}{l}\text { Antifungal } \\
\text { therapy }\end{array}$ & & & 0.1246 & & & & 0.1704 & \\
\hline \multicolumn{9}{|l|}{$\begin{array}{l}\text { at diagnosis, no. } \\
(\%)\end{array}$} \\
\hline Ployene & 0 & 1 & & & 2 & 3 & & \\
\hline Voriconazole & 7 & 10 & & & 0 & 5 & & \\
\hline Oral & 5 & 1 & & & 1 & 0 & & \\
\hline \multicolumn{9}{|l|}{ Itraconazole } \\
\hline Caspofungin & 1 & 4 & & & 1 & 1 & & \\
\hline $\begin{array}{l}\text { Surgical } \\
\text { intervention, no. } \\
(\%)\end{array}$ & 5 & 0 & 0.0056 & 0.99 & 4 & 3 & 0.1447 & \\
\hline
\end{tabular}

Abbreviation: IPA: invasive pulmonary aspergillosis; PM: pulmonary mucormycosis; APACHE II: Acute Physiology and Chronic Health Evaluation II; RF: respiratory failure.

\section{Figures}




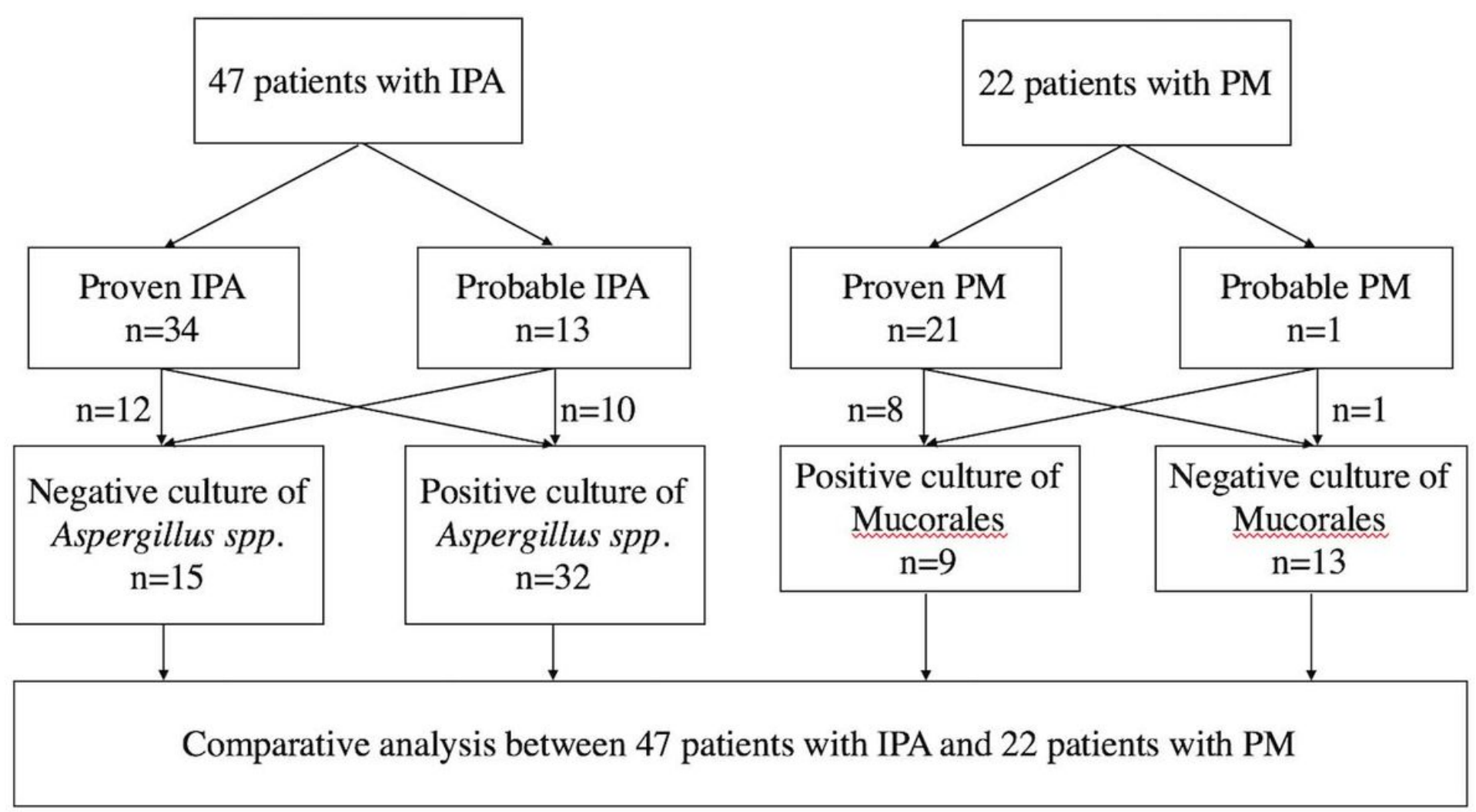

Figure 1

Schematic flow chart of the study. IPA, invasive pulmonary aspergillosis; PM, pulmonary mucormycosis.
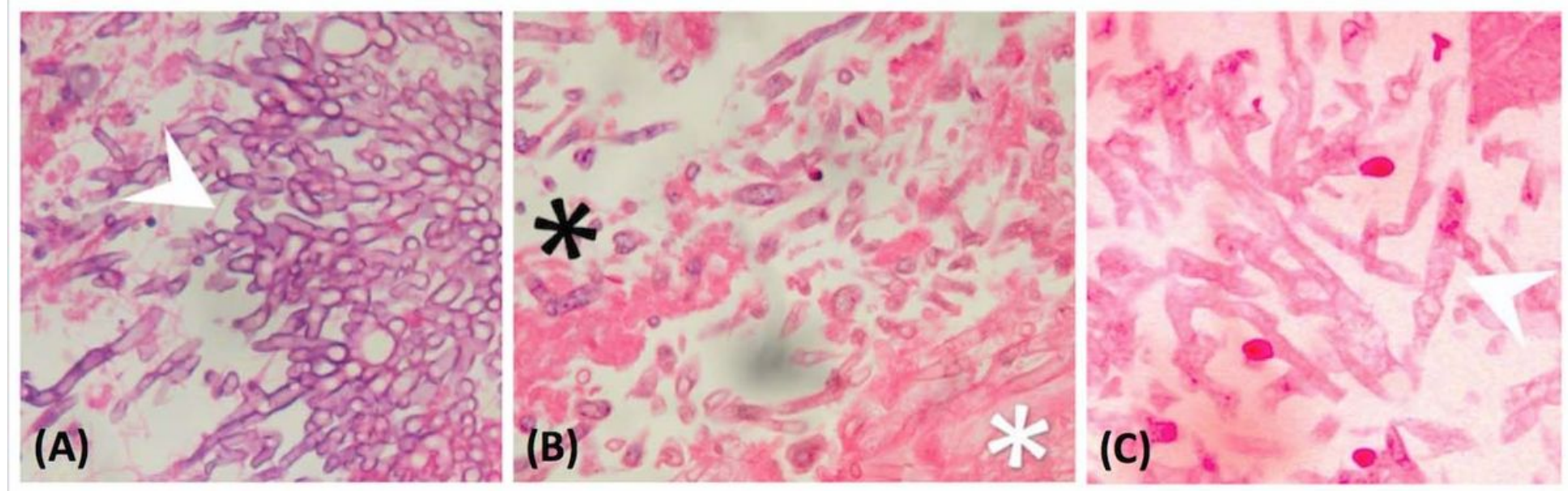

\section{Figure 2}

Histopathological finding (A) Septate fungal hyphae branching at a $45^{\circ}$ angle (arrowhead), which is characteristic of Aspergillus spp. (magnification: 400x), (B) Broad-based, aseptate hyphae, which are characteristic of Mucormycete (white star) and the other septate fungal hyphae are Aspergillus spp. (black star) (magnification: 400x), (C) Mucormycete characterized with broad-based, aseptate hyphae (arrowhead) (magnification: 400x). 

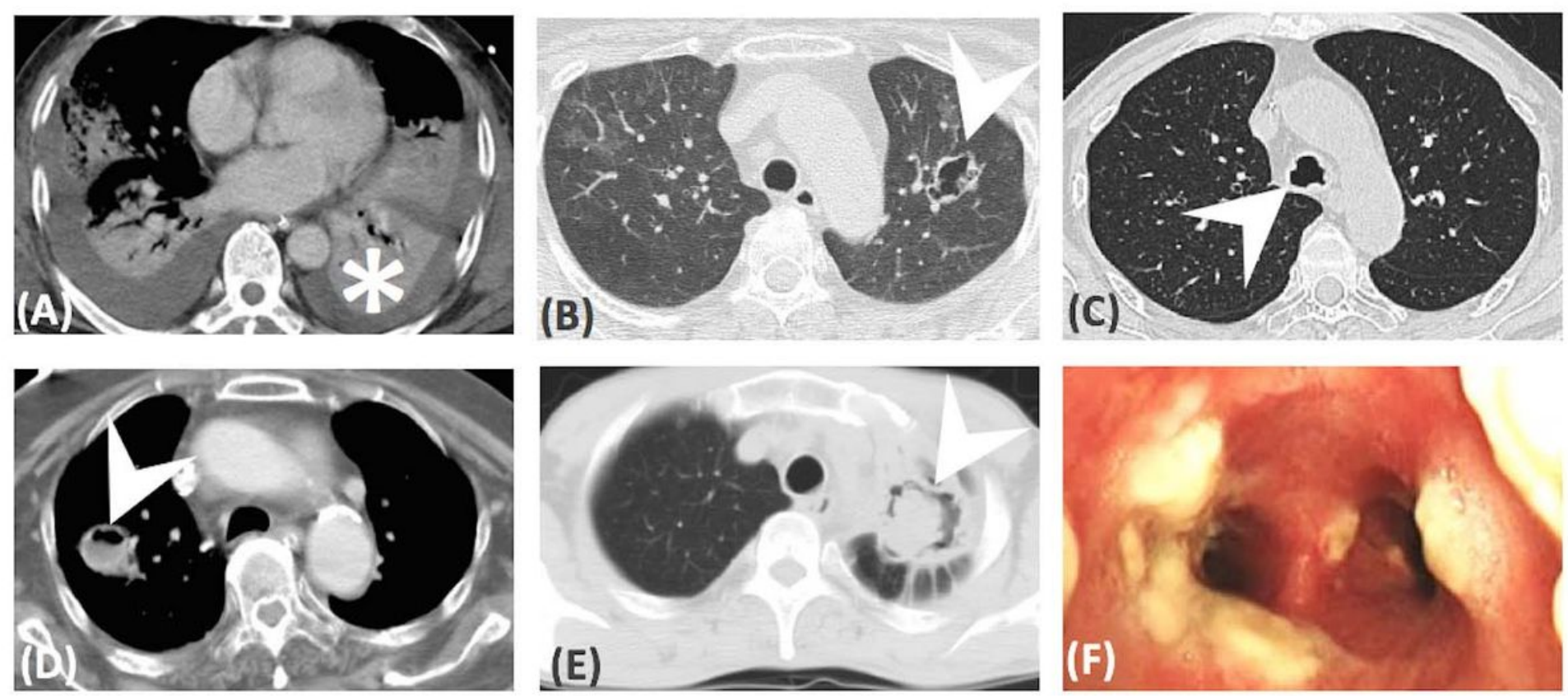

Figure 3

CT images (A) Consolidation, (B) Cavitation, (C) Abscess formation, (D) Ball in hole, (C, F) Airway involvement only. 